\title{
Auction-based allocation of shared electricity storage resources through physical storage rights
}

\author{
Tom Brijs ${ }^{\mathrm{a}, \mathrm{b}, \mathrm{d}, *}$, Daniel Huppmann ${ }^{\mathrm{c}, \mathrm{d}}$, Sauleh Siddiqui ${ }^{\mathrm{d}, \mathrm{e}}$, Ronnie Belmans ${ }^{\mathrm{a}, \mathrm{b}}$ \\ ${ }^{a}$ Department of Electrical Engineering, University of Leuven (KU Leuven), Heverlee, Belgium \\ ${ }^{\mathrm{b}}$ EnergyVille Research Institute, Genk, Belgium \\ ${ }^{\mathrm{c}}$ International Institute of Applied Systems Analysis (IIASA), Laxenburg, Austria \\ ${ }^{\mathrm{d}}$ Department of Civil Engineering, The Johns Hopkins University, Baltimore, MD, USA \\ e Department of Applied Mathematics and Statistics, The Johns Hopkins University, Baltimore, MD, USA
}

\section{A R T I C L E I N F O}

\section{Article history:}

Received 12 March 2016

Received in revised form 13 May 2016

Accepted 17 May 2016

Available online

\section{Keywords:}

Electricity storage

Shared storage resources

Auction-based allocation

(Generalized) Nash equilibrium

Mixed complementarity problem

\begin{abstract}
A B S T R A C T
This article proposes a new electricity storage business model based on multiple simultaneously considered revenue streams, which can be attributed to different market activities and players. These players thus share electricity storage resources and compete to obtain the right to use them in a dynamic allocation mechanism. It is based on the design of a new periodically organized auction to allocate shared storage resources through physical storage rights between different market players and accompanying applications. Through such a flexibility platform owners of flexible resources can commercialize their flexible capacity over different applications, while market players looking for additional flexibility can obtain this through a pay-per-use principle and thus not having to make long-term investment commitments. As such, they can quickly adapt their portfolio according to the market situation. Alternatively, through such an allocation mechanism players can effectively share storage resources. Players may be incentivized to participate as they can share the investment cost, mitigate risk, exploit economies of scale, overcome regulatory barriers, and merge time-varying and player-dependent flexibility needs. The mechanism allocates the limited storage resources to the most valuable application for each market-clearing, based on the competing players' willingness-to-pay. An illustrative case study is provided in which three players share storage resources that are allocated through a daily auction with hourly market-clearings.
\end{abstract}

(c) 2016 Elsevier Ltd. All rights reserved.

\section{Introduction}

The integration of variable renewable energy sources (RES) is a major challenge for the operation of the power system. Their limited controllability and predictability results in an increased need for power system flexibility, while flexible conventional power plants currently experience decreasing profitability as a result of low electricity prices and a limited number of operating hours [1]. Flexibility is the ability to provide up- and downward power adjustments to deal with temporary imbalances between generation and consumption of electric energy [2,3]. This flexibility can be provided by flexible generation and consumption, and

\footnotetext{
* Corresponding author at: Department of Electrical Engineering, University of Leuven (KU Leuven), Heverlee, Belgium. Tel.: +32 485826161.

E-mail addresses: tom.brijs@esat.kuleuven.be (T. Brijs),

huppmann@iiasa.ac.at (D. Huppmann), siddiqui@jhu.edu (S. Siddiqui),

ronnie.belmans@esat.kuleuven.be (R. Belmans).
}

electricity storage, but can also be activated in neighboring regions through interconnection capacity and the further integration of adjacent markets (Fig. 1). Electricity storage has the ability to compensate temporary power surpluses and shortages by decoupling the generation of electric energy from its consumption over time. The extent of this compensation is limited by its storage capacity.

Although there is a need for flexibility because of its increasing demand and decreasing supply, market participants are only incentivized to integrate new flexible resources if the investment is profitable. In addition, the value of storage is often underestimated due to the focus on operation strategies based on only a single application, usually price arbitrage between off-peak and on-peak hours. However, determining the true value of electricity storage will likely require the aggregation of multiple applications while accounting for the interdependence between potential revenue streams [4-6]. The value of individual applications cannot simply be added together, but need to be co-optimized since different storage services can conflict with each other [7]. 


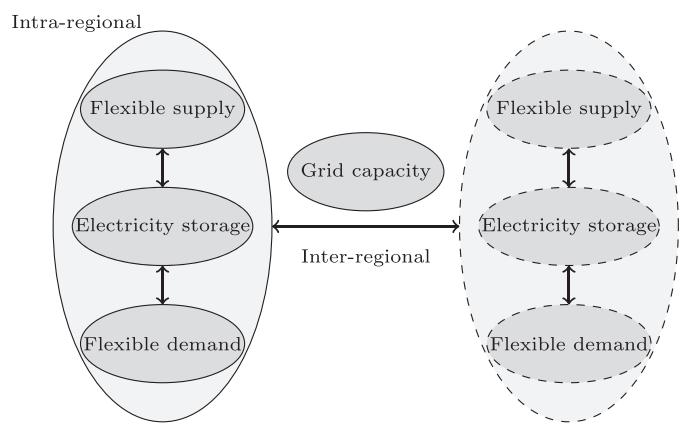

Fig. 1. Overview of power system flexibility sources.

Therefore, this article considers a new storage business model based on multiple simultaneously considered revenue streams, which can be attributed to different activities in the market and can thus be the focus of different market players. As such, these market players share electricity storage resources and compete to use the shared storage resources. The allocation is based on the design of a periodically organized auction with sequential marketclearings, in which the right to use storage resources is traded between different players.

\subsection{Electricity storage applications}

Electricity storage refers to systems, bidirectionally coupled with the power system, which buffer energy. This includes both systems in which the charging and discharging side is physically located at one location, e.g., pumped-hydro storage plants and battery storage systems, or at multiple locations, i.e., power-to-gas systems in combination with a gas turbine. This definition distinguishes electricity storage from the broader concept of energy storage, which may, e.g., also include stock-piling fuel at the supply side of the power system. ${ }^{1}$

Historically, electricity storage plants were considered as an alternative for investing in peak-load generation, by charging during off-peak and discharging during on-peak moments. However, due to the liberalization of electricity markets and the integration of RES, distinct valorization paths for different applications of storage emerged [8-11]. These can be categorized in energy, network, and reliability services.

Energy services include arbitrage and portfolio optimization of market participants. Arbitrage is based on price differences over time: electricity is bought and stored when the price is low, and is sold and generated again when the price is higher. Portfolio optimization is performed at different time scales, i.e., investment, scheduling, and operation, and covers generation investment deferral, inter-temporal energy shifting, and capacity firming, respectively. Through inter-temporal energy shifting generators optimize the value of generation by decoupling generation and physical injection, while consumers optimize the cost of consumption by decoupling consumption and physical withdrawal. Capacity firming can indicate the ability to smoothen the generation or consumption output, resulting in less volatile power profiles, or to follow predetermined output schedules to reduce imbalanced positions in real-time. Network services include the provision of frequency control (i.e., primary, secondary, tertiary), ${ }^{2}$

\footnotetext{
${ }^{1}$ Power plants may have significant fuel reserves, e.g., the natural gas grid with its storage capabilities for gas-fired power plants, coal piles at classic thermal power plants, and nuclear fuel at nuclear power plants.

${ }^{2}$ In the ENTSO-E synchronous zone operating reserves are categorized into frequency containment reserves (FCR), frequency restoration reserves (FRR), both automatic (aFRR) and manual (mFRR), and restoration reserves (RR).
}

voltage support, congestion management, and black-start capabilities to the transmission system operator (TSO). In the future, some of these will likely be provided to the distribution system operator (DSO) as well. Reliability services include the provision of reliability on both the local and system level.

This multitude of applications makes electricity storage plants an interesting asset for a wide range of market participants. However, operating a storage plant to provide just one or a few of these services might not always result in a positive business case; profitability may require the aggregation of multiple applications.

\subsection{Motivation}

Although some studies focus on the co-optimization of different storage applications (e.g., [5,7,12]), most existing work focuses on only a single application or allocates the available storage resources a-priori when considering multiple applications, instead of applying a periodically performed optimization process. In addition, the sharing and operation of storage resources by different players has only been studied to a limited extent, except for the work done by [4]. As such, the contribution of the auctionbased allocation described in this article is that it does not a-priori define the applications or even the market player that the storage resources will serve at a certain moment in time. This can be accomplished by the development of a centralized platform where periodical auctions with sequential market-clearings take place to allocate the right to use (dis)charge power capacities and energy storage capacity. These auctions can serve both settings where (1) multiple players share common storage plants and (2) multiple suppliers of storage resources and prospective consumers meet to trade physical storage rights. Whereas the presented allocation mechanism allows to simultaneously include multiple resource suppliers and players competing for the right to use them, and to simultaneously consider their offers, the method discussed in [4] considers a sequential allocation to players which express their need for flexible resources at different time scales. In addition, the presented allocation mechanism auctions physical (dis)charge power rights and storage capacity rights, whereas the allocation in [4] is based on actual utilization profiles.

Market players can have multiple incentives to share, contract, or offer storage resources by means of a periodically organized auction. First, this may allow them to exploit economies of scale, i.e., increasing the plant size at a reduced cost per unit of power and energy. Second, they can share the investment cost and associated risk, especially when considering large-scale storage plants. Third, as flexibility needs vary throughout the year and even throughout the day, and across market players, they may have different (possibly complementary) storage utilization patterns, providing an incentive to share resources.

From a system point of view, there are additional reasons to share storage resources. First, as storage resources are usually limited due to geographical requirements, they should be allocated to the most valuable services at each point in time. Second, due to the introduced competition to use storage resources strategic under- or overusage [13] is likely to occur less frequently. Third, although pumped-hydro storage is currently the most mature storage technology, rapidly decreasing costs and technological advancements are making battery storage systems increasingly competitive [14]. To overcome barriers for such small-scale storage resources to participate in the market, the development of a centralized platform allows owners of these resources to offer flexibility to market players that aggregate them. Finally, regulatory barriers might prevent storage operators to provide certain services simultaneously. In the United States storage plants can either provide market-based or regulated services (e.g., congestion management to avoid grid upgrades), but they are 
not allowed to combine both in a single business case [15]. An auction such as the one proposed in this article can overcome this regulatory barrier by allocating storage rights to different players to provide either market-based or regulated services.

This decoupling of the ownership of storage resources with its physical operation has similar characteristics to the treatment of transmission capacity, as both have the ability to move power, the former in time while the latter in space. In European electricity markets cross-border transmission capacity is auctioned explicitly or implicitly $[16,17]$. The former indicates that market players can obtain the right to use interconnector capacity, after which they can use these capacities to capture price differences in neighboring markets. In the latter, these capacities are not auctioned to market players but allocated to the power exchange to include in the market-clearing algorithm to maximize social welfare. The allocation mechanism discussed in this article is based on explicit auctioning, as first the right to use storage resources is auctioned, after which players can use these resources in the electricity market. Furthermore, Refs. $[18,19]$ consider a situation where the surplus collected by the system operator or power exchange (i.e., storage congestion rent), following a central operation of storage resources to maximize social welfare, is allocated to players holding financial storage rights. These are based on the design of financial transmission rights [20,21], and thus remunerate storage investors by either the revenues of the auction of financial storage rights or the value of the storage congestion rent itself. Similar to the proposed auction-based allocation mechanism, this allows them to recover the investment cost without participating in the electricity market themselves.

\subsection{Contributions}

The main contribution of this article is the presentation of an alternative approach for electricity storage plants to aggregate multiple applications. This is based on a new market for flexibility, namely a periodically organized auction to allocate shared storage resources through physical storage rights between different market players and accompanying applications. Through this allocation mechanism (1) market participants can share storage resources to exploit economies of scale, reduce the investment cost, mitigate risk, match complementary flexibility needs, and overcome regulatory barriers, and (2) owners of flexible resources can commercialize their flexible capacity over different applications while market players looking for additional flexibility have access to these resources on a short-term basis. As such, the latter do not have to make long-term investment commitments and can adapt their portfolio according to changing market situations.

The article is structured as follows. Section 2 discusses (Generalized) Nash games, mixed complementarity problems, and the designed storage allocation mechanism in more detail. Section 3 illustrates this auction-based allocation through a case study in which three market players share storage resources, by providing the mathematical formulation of the players' individual optimization problems and resulting market equilibrium problem. While Section 4 discusses the case study's results, Section 5 provides the conclusions of this article.

\section{Methodology}

\section{1. (Generalized) Nash equilibrium problems}

The interaction between several market players, in which each player aims to optimize the value of its objective function given the decisions by all rivals, can be mathematically formulated as an equilibrium problem. We first introduce Nash equilibrium problems (NEP) [22] before discussing the concept of a

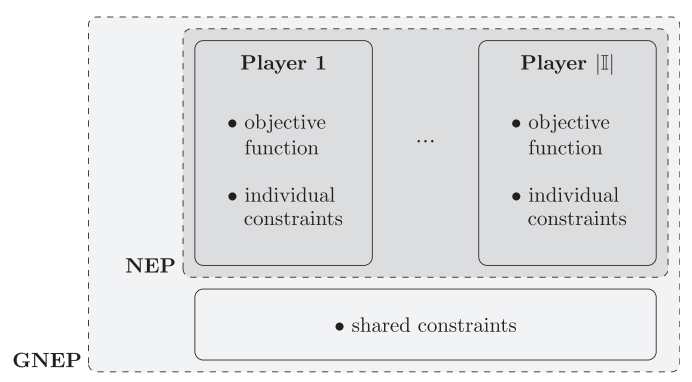

Fig. 2. Illustration of (Generalized) Nash equilibrium problems.

Generalized Nash equilibrium problem (GNEP) [23]. Assume a market with a finite amount of players, in which each player $i \in \mathbb{}$ faces the following optimization problem:

$\max _{x_{i}} f_{i}\left(x_{i}, x_{-i}\right)$

s.t. $x_{i} \in \mathbb{X}_{i}$.

Each player's vector of decision variables $x_{i}$, has to be chosen from its set of feasible strategies $\mathbb{X}_{i}$, while the vector of decision variables of its rivals $x_{-i}$ is considered as given. A Nash equilibrium $x_{i}^{*}$ is then reached when the following condition holds:

$f_{i}\left(x_{i}^{*}, x_{-i}^{*}\right) \geq f_{i}\left(y_{i}, x_{-i}^{*}\right), \quad \forall i \in \mathbb{Q}, y_{i} \in \mathbb{X}_{i}$.

This equilibrium means that given the decisions by all rivals, no player has an incentive to deviate from its chosen strategy. An implicit assumption of the NEP is that the strategies chosen by the competing players only affect the players' objective function and not their feasible set of strategies. In contrast, in a GNEP this assumption is relaxed [23-27], as each player's vector of decision variables $x_{i}$ has to be chosen from a set of feasible strategies $\mathbb{X}_{i}\left(x_{-i}\right)$ that is affected by the strategies chosen by the competing players. A Generalized Nash equilibrium $x_{i}^{*}$ is then reached when the following condition holds:

$f_{i}\left(x_{i}^{*}, x_{-i}^{*}\right) \geq f_{i}\left(y_{i}, x_{-i}^{*}\right), \quad \forall i \in \mathbb{Q}, y_{i} \in \mathbb{X}_{i}\left(x_{-i}^{*}\right)$.

The general structure of both a NEP and GNEP, consisting of a set of interrelated optimization problems, is illustrated in Fig. 2. In a GNEP, each player's objective function may be subject to both individual and shared constraints. While each individual optimization problem represents the decision process of one player, the equilibrium problem represents the interactions in a market environment of multiple interrelated players.

\subsection{Mixed complementarity problems}

The NEP and GNEP can be solved by formulating the problem as a mixed complementarity problem (MCP). This is done by deriving the first-order optimality, or Karush-Kuhn-Tucker (KKT), conditions of each player's optimization problem and solving them simultaneously. In the MCP formulation, the complementarity conditions enforce that the inner product of an inequality constraint and the primal or dual variable ${ }^{3}$ is zero, and the nonnegativity of both the inequality constraint and the primal or dual variable. This means that either the inequality constraint holds as an equality, i.e., is binding, or the primal or dual variable is

\footnotetext{
${ }^{3}$ A constraint's dual variable represents the incremental improvement of the player's objective value when marginally relaxing the respective constraint, and can be interpreted as the marginal price of the resource subject to the constraint.
} 


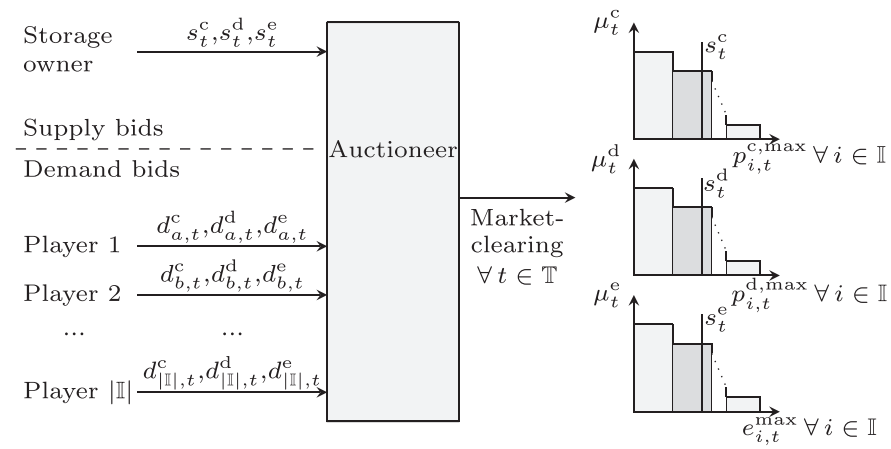

Fig. 3. Illustration of the market-clearing mechanism to allocate storage resources.

zero. Mathematically, this is expressed by using the perpendicular operator $\perp$, which indicates complementarity.

An MCP is thus an array of equalities and inequalities which is obtained by aggregating all players' KKT conditions. However, when tackling a GNEP, aggregating the individual players' KKT conditions into an MCP results in a nonsquare system: the shared constraints are identical for each player, while the associated dual variables of each involved player may hold different values. This 'squareness' issue can be solved by assigning an identical dual variable for each player to the shared constraint [23], meaning that each player values the shared resource identically, which leads to a single 'price' for the shared resource [24,25].

This approach can be interpreted as an auctioneer allocating the shared resource to the players according to the price they are willing to pay to obtain the right to use it. Their willingness-to-pay directly relates to the improvement of their objective value from a marginal relaxation of the shared resource.

\subsection{Auction-based allocation of shared storage resources}

The shared storage resources' allocation problem can be formulated as both a NEP and GNEP. In the former case, the suppliers of storage resources are modeled explicitly, while the consumers and suppliers in the market for storage resources interact by means of market-clearing conditions representing the auctioneer. This formulation relates to the situation where multiple suppliers and consumers of storage resources compete in a centralized market. In the latter case, the suppliers are not modeled explicitly but are included implicitly through the storage resources' shared constraints. Through these shared constraints an auctioneer is assumed to allocate the storage rights over the different players. This formulation is particularly useful to represent the situation where multiple market players share the storage resources and allocate them periodically among each other.

It is well known from [23] that a NEP where the auctioneer is modeled explicitly yields the same solution as a GNEP where the dual variables of each player for the shared constraints are assumed to be identical. If the solution is nonunique, the two solutions may differ in terms of the primal variables (i.e., operation decisions), but the objective value (i.e., pay-off) for each player must be identical. In this article, we use the GNEP formulation to a case study in Section 3 as it illustrates a case in which three players share storage resources. For illustrative purposes, the remainder of the discussion assumes a single storage plant.

In both formulations the auctioneer thus acts as a facilitator between the supply of shared storage resources (i.e., charge power $P^{c, m a x}$, discharge power $P^{\mathrm{d}, \max }$, energy storage capacity $\left.E^{\max }\right)^{4}$ and a

\footnotetext{
${ }^{4}$ Table 1 provides an overview of the symbols for sets, primal variables, dual variables, and parameters used in this article. Formulas are provided assuming SI or base units, while input data and results follow typical units in electrical engineering.
}

number of players $\mid$ | $\mid$ which compete to obtain the right to use them. A periodical auction is organized, in which for each market period $t \in \mathbb{T}$ the supplier of the storage resources submits supply bids $s_{t}^{\mathrm{c}}, s_{t}^{\mathrm{d}}, s_{t}^{\mathrm{e}}$ and each market player $i \in \mathbb{}$ has the opportunity to submit demand bids $d_{i, t}^{c}, d_{i, t}^{\mathrm{d}}, d_{i, t}^{\mathrm{e}}$ for each storage resource (Fig. 3 , left). The supplier is assumed to provide a supply bid for each shared resource equal to its maximum capacity, to be sold at any price defined by the market. Each player $i$ bids the maximum price he is willing to pay to obtain the right to use a specified volume of each storage resource. This maximum price equals its incremental pay-off. The auctioneer then aggregates the demand bids for each storage resource, i.e., the demand curve, and matches them with each resource's supply bid, i.e., the supply curve, which results in a market-clearing for each timestep $t$ (Fig. 3, right). This yields a cleared volume for the charge power rights $p_{i, t}^{\text {c,max }}$, discharge power rights $p_{i, t}^{\mathrm{d}, \max }$, and storage capacity rights $e_{i, t}^{\max }$ for each player $i$, and uniform market-clearing prices $\mu_{t}^{\mathrm{c}}, \mu_{t}^{\mathrm{d}}, \mu_{t}^{\mathrm{e}}$. In equilibrium, these prices equal the marginal willingness-to-pay for each respective resource.

Similar to the case in current electricity markets, the allocation process may be iterated at different timeframes (e.g., week-ahead, day-ahead, intra-day, real-time) to allow players to adjust their obtained physical storage rights, based on updated market information. In a first auction (e.g., day-ahead) the shared resources are allocated to the different players according to their willingness-to-pay, which is dependent on their market expectations and risk aversion, while in a consecutive allocation closer to real-time (e.g., intra-day) players can trade and reallocate the obtained resources among each other: players that contracted too much can offer part of their obtained rights again to the platform, while players that contracted too little can bid to obtain additional storage rights. As is the case in electricity markets, these sequential markets for storage resources may also allow for arbitrage opportunities as price spreads can be captured over the different sequential markets. Arbitrageurs could, e.g., obtain additional storage rights at the day-ahead stage to afterwards sell in the intra-day market to other players if they expect the intra-day price to clear at a higher price. Alternatively, players could, e.g., postpone the reservation of physical storage rights in the day-ahead market to the intra-day market if they expect the intra-day market to clear at a lower price. Although the value of this arbitrage is related to the presence of price spreads, it also depends on the effect of additional/fewer requested storage rights on prices, as arbitrage may reduce price spreads by increasing low prices when requesting additional storage rights and decreasing high prices when requesting fewer storage rights.

\section{Case study}

To illustrate the presented auction-based allocation mechanism, a case study is shown for a daily auction with hourly 
Table 1

Table of symbols.

\begin{tabular}{|c|c|c|c|c|}
\hline Type & Symbol & Quantity & Unit (SI) & Typical unit \\
\hline \multirow[t]{3}{*}{ Sets } & $h \in \mathbb{U}$ & Time steps & - & - \\
\hline & $i \in \mathbb{\square}$ & Players & - & - \\
\hline & $t \in \mathbb{T}$ & Time stamps & - & - \\
\hline \multirow[t]{11}{*}{ Primal variables } & $e_{i, h}$ & Stored energy & $\mathrm{J}$ & MWh \\
\hline & $e_{i}^{\max }, e_{i h}^{\max }, e_{i, t}^{\max }$ & Storage capacity rights & $\mathrm{J}$ & MWh \\
\hline & $p_{i h}^{c}$ & Charge power & $\mathrm{W}$ & MW \\
\hline & $p_{i}^{\mathrm{c}, \mathrm{max}}, p_{i, h}^{\mathrm{c}, \max }, p_{i, t}^{\mathrm{c}, \max }$ & Charge power rights & $\mathrm{W}$ & MW \\
\hline & & Discharge power & $\mathrm{W}$ & MW \\
\hline & $p_{i}^{\mathrm{d}, \mathrm{hax}}, p_{i, h}^{\mathrm{d}, \max }, p_{i}^{\mathrm{d}, \text { max }}$ & Discharge power rights & $\mathrm{W}$ & MW \\
\hline & $p_{j h}^{g}$ & Power generation & $\mathrm{W}$ & MW \\
\hline & $p_{i h}^{1, h}$ & Power curtailment & $\mathrm{W}$ & MW \\
\hline & $\beta_{i}^{i, h}$ & Cost & $€ / s$ & $€ /$ year \\
\hline & $\pi_{i}$ & Profit & $€ / s$ & $€ /$ year \\
\hline & $\pi_{i}^{\mathrm{op}}$ & Operating profit & $€ / s$ & $€ /$ year \\
\hline \multirow[t]{15}{*}{ Dual variables } & $\mu_{i, h}^{\mathrm{c}}$ & Dual to charge power constraints & $€ / \mathrm{s} / \mathrm{W}$ & $€ / \mathrm{h} / \mathrm{MW}$ \\
\hline & $\mu_{h}^{\mathrm{c}, h}, \mu_{t}^{\mathrm{c}}$ & Price of charge power rights & $€ / \mathrm{s} / \mathrm{W}$ & $€ / \mathrm{h} / \mathrm{MW}$ \\
\hline & $\mu^{\mathrm{c}}$ & Price of charge power rights & $€ / s / \mathrm{W}$ & $€ /$ day/MW \\
\hline & $\mu_{i, h}^{\mathrm{d}}$ & Dual to discharge power constraints & $€ / \mathrm{s} / \mathrm{W}$ & $€ / \mathrm{h} / \mathrm{MW}$ \\
\hline & $\mu_{h}^{\mathrm{d}}, \mu_{t}^{\mathrm{d}}$ & Price of discharge power rights & $€ / s / \mathrm{W}$ & $€ / \mathrm{h} / \mathrm{MW}$ \\
\hline & $\mu^{\mathrm{d} p t}$ & Price of discharge power rights & $€ / s / \mathrm{W}$ & $€ /$ day/MW \\
\hline & $\mu_{i h}^{\mathrm{e}}$ & Dual to storage capacity constraints & $€ / s / J$ & $€ / \mathrm{h} / \mathrm{MWh}$ \\
\hline & $\mu_{h}^{\mathrm{e}}, \mu_{t}^{\mathrm{e}}$ & Price of storage capacity rights & $€ / s / J$ & $€ / \mathrm{h} / \mathrm{MWh}$ \\
\hline & $\mu^{\mathrm{e}}$ & Price of storage capacity rights & $€ / s / J$ & $€ /$ day/MWh \\
\hline & $\gamma_{i, h}^{\mathrm{e}}$ & Dual to energy buffer constraints & $€ / s / J$ & $€ / \mathrm{h} / \mathrm{MWh}$ \\
\hline & $\gamma_{i, h}^{g}$ & Dual to available renewable power constraints & $€ / \mathrm{s} / \mathrm{W}$ & $€ / \mathrm{h} / \mathrm{MW}$ \\
\hline & $\gamma_{i, h}^{1}$ & Dual to imbalanced position constraints & $€ / s / W$ & $€ / \mathrm{h} / \mathrm{MW}$ \\
\hline & $\tau_{i, h}^{c}$ & Dual to charge power rights constraints & $€ / s / \mathrm{W}$ & $€ / \mathrm{h} / \mathrm{MW}$ \\
\hline & $\tau_{i h}^{\mathrm{d}}$ & Dual to discharge power rights constraints & $€ / s / \mathrm{W}$ & $€ / \mathrm{h} / \mathrm{MW}$ \\
\hline & $\tau_{i, h}^{\mathrm{e}, h}$ & Dual to storage capacity rights constraints & $€ / s / J$ & $€ / \mathrm{h} / \mathrm{MWh}$ \\
\hline \multirow[t]{9}{*}{ Parameters } & $E^{\max }$ & Storage capacity & $\mathrm{J}$ & MWh \\
\hline & $G_{p, h}$ & Available renewable power & W & MW \\
\hline & $L_{r}^{\max }$ & Imbalanced position upper bound & $\mathrm{W}$ & MW \\
\hline & $P^{c, \max }$ & Charge power rating & $\mathrm{W}$ & MW \\
\hline & $P^{\mathrm{d}, \max }$ & Discharge power rating & $\mathrm{W}$ & MW \\
\hline & $T^{\mathrm{h}}$ & Time step length & $\mathrm{s}$ & $\mathrm{h}$ \\
\hline & $\eta^{\mathrm{c}}, \eta^{\mathrm{d}}$ & (Dis)charge efficiency & $\%$ & $\%$ \\
\hline & $\lambda_{h}^{\mathrm{da}}$ & Day-ahead price & $€ / J$ & $€ / M W h$ \\
\hline & $\lambda_{h}^{\mathrm{rt}}$ & Real-time imbalance price & $€ / J$ & $€ / \mathrm{MWh}$ \\
\hline
\end{tabular}

market-clearings in which three market players compete for constrained storage resources, i.e., $\mathbb{\square}=\{a, p, r\}$, with index $a$ representing a player arbitraging day-ahead market prices, index $p$ a player focusing on portfolio management, and index $r$ a player that aims to use storage resources to capture imbalance price differences in the real-time market. First, the individual optimization problems are presented as if electricity storage resources would be readily available to them. Second, we discuss which changes have to take place in order for the players to share storage resources and compete in an auction-based allocation mechanism, i.e., equilibrium problem. Third, the MCP formulation of the equilibrium problem is discussed while it is provided in full in Appendix A.

The model formulations of the provided case study include discretized hourly time periods $h \in \mathbb{M}$, with $|\mathbb{M}|=24$ and $T^{\mathrm{h}}$ representing the length of one time period, i.e., one hour. Variables in parentheses denote the dual variables of the respective constraints. In addition, all players are assumed to be price-takers with perfect foresight for the next optimization horizon, i.e., the next day in this case study. The storage plant is assumed to have sufficiently fast ramp rates for the considered hourly time resolution, with no restrictions regarding simultaneous charge and discharge actions, and a sufficiently large cycle-life such that its impact on the operation is negligible. These storage plant assumptions might serve to model typical pumped-hydro storage plants.

\subsection{Individual optimization problems}

First a storage operator is considered that aims to capture price differences in the day-ahead market. This player is indicated by index $a$, and its optimization problem, in which the pay-off is maximized over a time horizon $|\mathbb{H}| \cdot T^{\mathrm{h}}$, reads as follows:

$$
\begin{aligned}
& \max _{e_{a, h}, p_{a, h}^{\mathrm{c}}, p_{a, h}^{\mathrm{d}}} \sum_{h \in \mathbb{U}} \lambda_{h}^{\mathrm{da}} \cdot\left[T^{\mathrm{h}} \cdot\left(p_{a, h}^{\mathrm{d}}-p_{a, h}^{\mathrm{c}}\right)\right] /\left(|\mathbb{H}| \cdot T^{\mathrm{h}}\right), \\
& e_{a, h}=e_{a, h-1}+T^{\mathrm{h}} \cdot\left(p_{a, h}^{\mathrm{c}} \cdot \eta^{\mathrm{c}}-p_{a, h}^{\mathrm{d}} / \eta^{\mathrm{d}}\right), \quad\left(\gamma_{a, h}^{\mathrm{e}}\right), \quad \forall h \in \mathbb{U}, \\
& p_{a, h}^{\mathrm{c}} \leq P^{\mathrm{c}, \max }, \quad\left(\mu_{a, h}^{\mathrm{c}}\right), \quad \forall h \in \mathbb{U}, \\
& p_{a, h}^{\mathrm{d}} \leq P^{\mathrm{d}, \max }, \quad\left(\mu_{a, h}^{\mathrm{d}}\right), \quad \forall h \in \mathbb{U}, \\
& e_{a, h} \leq E^{\mathrm{max}}, \quad\left(\mu_{a, h}^{\mathrm{e}}\right), \quad \forall h \in \mathbb{U}, \quad \\
& e_{a, h}, p_{a, h}^{\mathrm{c}}, p_{a, h}^{\mathrm{d}} \in \mathbb{R}_{+}, h \in \mathbb{N}, \quad \forall h \in \mathbb{U}
\end{aligned}
$$

with $p_{a, h}^{\mathrm{c}}$ the charge power, $p_{a, h}^{\mathrm{d}}$ the discharge power, $e_{a, h}$ the stored energy, $\lambda_{h}^{\mathrm{da}}$ the day-ahead market price, $\eta^{\mathrm{c}}$ the charge efficiency, and $\eta^{\mathrm{d}}$ the discharge efficiency. Constraint (4b) expresses the intertemporal character of electricity storage, 
while (4c)-(4e) represent capacity bounds on the electricity storage resources.

Next, a RES generator operating a portfolio of both wind and photovoltaic (PV) capacity is considered. This player uses storage resources to increase the market value of its RES generation. This can be done by either directly selling its RES power output to the market or temporarily storing it during low price periods. This application of electricity storage results from the fact that periods experiencing high RES generation often coincide with lower price periods [1]. This player is indicated by index $p$, and its optimization problem is:

$$
\begin{aligned}
& \max _{\substack{p, h \\
e_{p, h}^{\mathrm{c}}, p_{p, h}^{\mathrm{d}},}} \sum_{h \in \mathbb{H}} \lambda_{h}^{\mathrm{da}} \cdot\left[T^{\mathrm{h}} \cdot\left(p_{p, h}^{\mathrm{g}}+p_{p, h}^{\mathrm{d}}\right)\right] /\left(|\mathbb{H}| \cdot T^{\mathrm{h}}\right), \\
& p_{p, h}^{\mathrm{g}}, p_{p, h}^{\mathrm{l}} \\
& p_{p, h}^{\mathrm{c}}+p_{p, h}^{\mathrm{g}}+p_{p, h}^{\mathrm{l}}=G_{p, h}, \quad\left(\gamma_{p, h}^{\mathrm{g}}\right), \quad \forall h \in \mathbb{H}, \\
& e_{p, h}=e_{p, h-1}+T^{\mathrm{h}} \cdot\left(p_{p, h}^{\mathrm{c}} \cdot \eta^{\mathrm{c}}-p_{p, h}^{\mathrm{d}} / \eta^{\mathrm{d}}\right), \quad\left(\gamma_{p, h}^{\mathrm{e}}\right), \quad \forall h \in \mathbb{U}, \\
& p_{p, h}^{\mathrm{c}} \leq P^{\mathrm{c}, \max }, \quad\left(\mu_{p, h}^{\mathrm{c}}\right), \quad \forall h \in \mathbb{H}, \\
& p_{p, h}^{\mathrm{d}} \leq P^{\mathrm{d}, \max }, \quad\left(\mu_{p, h}^{\mathrm{d}}\right), \quad \forall h \in \mathbb{H}, \\
& e_{p, h} \leq E^{\mathrm{max}}, \quad\left(\mu_{p, h}^{\mathrm{e}}\right), \quad \forall h \in \mathbb{H},
\end{aligned}
$$

$e_{p, h}, p_{p, h}^{\mathrm{c}}, p_{p, h}^{\mathrm{d}}, p_{p, h}^{\mathrm{g}}, p_{p, h}^{\mathrm{l}} \in \mathbb{R}_{+}, h \in \mathbb{N}, \quad \forall h \in \mathbb{U}$

with $G_{p, h}$ the available RES power output, $p_{p, h}^{g}$ the RES output directly sold to the market, and $p_{p, h}^{1}$ the curtailed RES output. Constraint ( $5 b$ ) denotes that the RES power output can either be stored, sold, or curtailed.

Unforeseen imbalances between generation and consumption are dealt with in real-time on the balancing market, which is coordinated by the TSO. At the procurement side of the balancing market the TSO contracts and activates reserve capacity to cover system imbalances, while at the settlement side of the balancing market the TSO settles individual imbalanced positions of market participants by means of an imbalance price that is based on the activation cost of reserves [1]. The third considered player is an arbitrageur that is active on the settlement side of the real-time balancing market to capture imbalance price differences over time. As the real-time balancing market is characterized by a small volume compared to the day-ahead market, the imbalanced positions this player can take while not diminishing the expected price spreads are assumed to be bounded by $L_{r}^{\max }(6 \mathrm{~b})$. As such, the price-taking assumption assumed in this illustrative case study holds. Although the balancing market is usually characterized by quarter-hourly or semi-hourly market periods, for illustrative purposes hourly market periods are assumed. This player is indicated by index $r$, and its optimization problem reads as follows:

$$
\begin{aligned}
& \max _{e_{r, h}, p_{r, h}^{\mathrm{c}}, p_{r, h}^{\mathrm{d}}} \sum_{h \in \mathbb{U}} \lambda_{h}^{\mathrm{rt}} \cdot\left[T^{\mathrm{h}} \cdot\left(p_{r, h}^{\mathrm{d}}-p_{r, h}^{\mathrm{c}}\right)\right] /\left(|\mathbb{H}| \cdot T^{\mathrm{h}}\right), \\
& p_{r, h}^{\mathrm{c}}+p_{r, h}^{\mathrm{d}} \leq L_{r}^{\max }, \quad\left(\gamma_{r, h}^{\mathrm{l}}\right), \quad \forall h \in \mathbb{U}, \\
& e_{r, h}=e_{r, h-1}+T^{\mathrm{h}} \cdot\left(p_{r, h}^{\mathrm{c}} \cdot \eta^{\mathrm{c}}-p_{r, h}^{\mathrm{d}} / \eta^{\mathrm{d}}\right), \quad\left(\gamma_{r, h}^{\mathrm{e}}\right), \quad \forall h \in \mathbb{U}, \\
& p_{r, h}^{\mathrm{c}} \leq P^{\mathrm{c}, \max }, \quad\left(\mu_{r, h}^{\mathrm{c}}\right), \quad \forall h \in \mathbb{U},
\end{aligned}
$$

$p_{r, h}^{\mathrm{d}} \leq P^{\mathrm{d}, \max }, \quad\left(\mu_{r, h}^{\mathrm{d}}\right), \quad \forall h \in \mathbb{U}$,

$e_{r, h} \leq E^{\max }, \quad\left(\mu_{r, h}^{\mathrm{e}}\right), \quad \forall h \in \mathbb{H}$,

$e_{r, h}, p_{r, h}^{\mathrm{c}}, p_{r, h}^{\mathrm{d}} \in \mathbb{R}_{+}, h \in \mathbb{N}, \quad \forall h \in \mathbb{M}$

with $\lambda_{h}^{\text {rt }}$ the real-time imbalance price.

\subsection{Generalized Nash equilibrium problem}

When formulating the presented optimization problems as a GNEP, two changes take place. First, as they compete for the shared electricity storage resources, the constraints representing the limited charge power (4c), (5d), (6d), discharge power (4d), (5e), (6e), and energy storage capacity (4e), (5f), (6f) are replaced by:

$p_{i, h}^{\mathrm{c}} \leq p_{i, h}^{\mathrm{c}, \max }, \quad\left(\tau_{i, h}^{\mathrm{c}}\right), \quad \forall i \in \mathbb{\mathbb { N }}, h \in \mathbb{U}$,

$p_{i, h}^{\mathrm{d}} \leq p_{i, h}^{\mathrm{d}, \max }, \quad\left(\tau_{i, h}^{\mathrm{d}}\right), \quad \forall i \in \mathbb{Q}, h \in \mathbb{U}$,

$e_{i, h} \leq e_{i, h}^{\max }, \quad\left(\tau_{i, h}^{\mathrm{e}}\right), \quad \forall i \in \mathbb{Q}, h \in \mathbb{U}$,

with $p_{i, h}^{\mathrm{c}, \max }, p_{i, h}^{\mathrm{d}, \max }$, and $e_{i, h}^{\max }$ the allocated charge power rights, discharge power rights, and storage capacity rights, respectively. These physical rights are bounded by the supplied storage resources, which are assumed to equal the installed (dis)charge power rating and energy storage capacity in this illustrative case study:

$$
\begin{aligned}
& \sum_{i \in \mathbb{Q}} p_{i, h}^{\mathrm{c}, \max } \leq P^{\mathrm{c}, \max }, \quad\left(\mu_{h}^{\mathrm{c}}\right), \quad \forall h \in \mathbb{U}, \\
& \sum_{i \in \mathbb{Q}} p_{i, h}^{\mathrm{d}, \max } \leq P^{\mathrm{d}, \max }, \quad\left(\mu_{h}^{\mathrm{d}}\right), \quad \forall h \in \mathbb{U}, \\
& \sum_{i \in \mathbb{Q}} e_{i, h}^{\max } \leq E^{\max }, \quad\left(\mu_{h}^{\mathrm{e}}\right), \quad \forall h \in \mathbb{U} .
\end{aligned}
$$

Alternatively, when considering a centralized market for storage resources rather than a situation where players share them, the supply is characterized by index $h$ as well as it will be time-varying. Second, a cost term is subtracted ex-post from each player's objective value, since the right to use the limited storage resources is now allocated through an auction instead of being readily available to them. The uniform price of the shared resources $\mu_{h}^{c}(8 \mathrm{a}), \mu_{h}^{\mathrm{d}}(8 \mathrm{~b}), \mu_{h}^{\mathrm{e}}(8 \mathrm{c})$ at each hourly marketclearing is determined by the willingness-to-pay of the players' marginally cleared demand bid to obtain the right to use them. The ex-post calculation of the profit $\pi_{i}$ as opposed to the operating profit $\pi_{i}^{\mathrm{op}}$ is done by considering the objective value resulting from (4a), (5a), (6a) and subtracting a cost term $\beta_{i}$ :

$\beta_{i}=\sum_{h \in \mathbb{U}}\left(p_{i, h}^{\mathrm{c}, \max } \cdot \mu_{h}^{\mathrm{c}}+p_{i, h}^{\mathrm{d}, \max } \cdot \mu_{h}^{\mathrm{d}}+e_{i, h}^{\max } \cdot \mu_{h}^{\mathrm{e}}\right), \quad \forall i \in \mathbb{\square}$,

$\pi_{i}^{\mathrm{op}}-\beta_{i}=\pi_{i}, \quad \forall i \in \mathbb{0}$.

The MCP comprised of each player's KKT conditions and the shared constraints is solved in GAMS using the PATH solver [28], and is provided in Appendix A for both a daily auction with hourly market-clearings, and a less dynamic periodically organized (e.g., daily, weekly) auction including a single market-clearing, i.e., allocation, for each of the shared resources for the entire period (e.g., day, week). Since the considered optimization problems are 
Table 2

Table of input parameters.

\begin{tabular}{|c|c|c|c|c|c|c|c|}
\hline$E^{\max }$ & $200 \mathrm{MWh}$ & $L_{c}^{\max }$ & $25 \mathrm{MW}$ & $P^{\mathrm{d}, \max }$ & $50 \mathrm{MW}$ & $\eta^{\mathrm{c}}$ & $86.6 \%$ \\
\hline$G_{p}^{\max }$ & $75 \mathrm{MW}+75 \mathrm{MW}$ & $P^{\mathrm{c}, \max }$ & $50 \mathrm{MW}$ & $T^{\mathrm{h}}$ & $1 \mathrm{~h}$ & $\eta^{\mathrm{d}}$ & $86.6 \%$ \\
\hline
\end{tabular}

Table 3

Yearly operating profit, cost to obtain storage rights, and profit, 2014.

\begin{tabular}{|c|c|c|c|c|c|c|}
\hline & \multicolumn{3}{|c|}{ Daily auctions with hourly allocations } & \multicolumn{3}{|c|}{ Daily auctions with daily allocations } \\
\hline & Operating profit & Cost & Profit & Operating profit & Cost & Profit \\
\hline & $\pi_{i}^{\mathrm{op}}$ & $\beta_{i}$ & $\pi_{i}$ & $\pi_{i}^{\mathrm{op}}$ & $\beta_{i}$ & \\
\hline & [M€/year] & [M€/year] & {$[\mathrm{M} € /$ year $]$} & {$[\mathrm{M} € /$ year $]$} & [M€/year] & [M€/year] \\
\hline Player $a$ & 0.445 & 0.445 & 0.000 & 0.365 & 0.365 & 0.000 \\
\hline Player $p$ & 0.369 & 0.336 & 0.033 & 0.230 & 0.197 & 0.033 \\
\hline Player $r$ & 1.934 & 0.367 & 1.567 & 1.958 & 0.584 & 1.374 \\
\hline$\sum_{i \in \mathbb{1}}$ & 2.748 & 1.148 & 1.600 & 2.553 & 1.146 & 1.407 \\
\hline
\end{tabular}

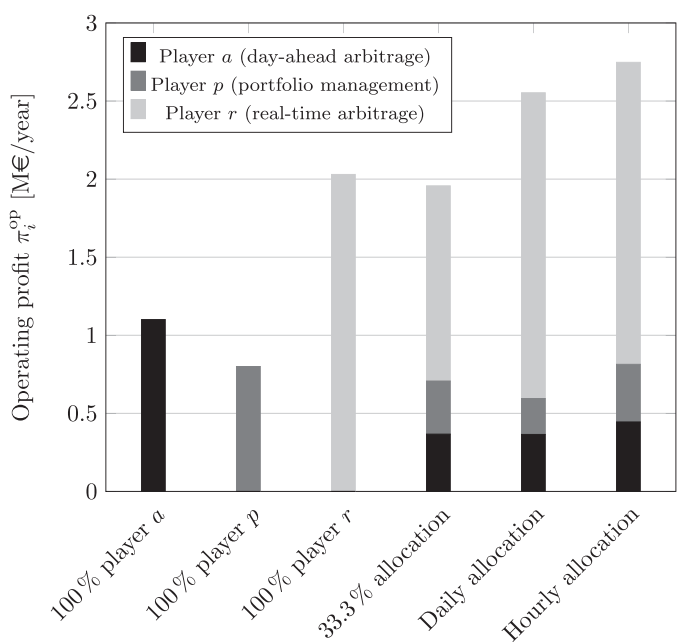

Fig. 4. Total and individual operating profit for different allocations of the shared storage resources, 2014.

convex and the players only face linear constraints, the KKT conditions are both necessary (i.e., an optimal solution satisfies the KKT conditions) and sufficient (i.e., each KKT point is an optimal solution) [27].

\section{Results}

The Belgian day-ahead market price [29], real-time imbalance price [30], and RES generation profiles [30] for 2014 are used for the illustrative case study. The hourly imbalance price $\lambda_{h}^{\text {rt }}$ is calculated as the average of the four quarter-hourly imbalance prices in hour $h$. The RES portfolio of player $p$ is assumed to consist of both PV systems and offshore wind turbines, both accounting for $50 \%$ of the portfolio. The time-varying available RES power output $G_{p, h}$ is determined by multiplying the hourly availability of the respective sources by the installed capacity $G_{p}^{\max }$. The storage plant characteristics used for the case study, along with other input data, are displayed in Table 2.

Fig. 4 shows the individual operating profit $\pi_{i}^{\text {op }}$ and total operating profit $\sum_{i \in \mathbb{1}} \pi_{i}^{\mathrm{op}}$ for 2014 as a result of the use of storage resources $^{5}$ for different allocations, either fixed a-priori defined allocations (i.e., columns 1-4) or allocations resulting from the

\footnotetext{
${ }^{5}$ This means that for player $p$ the value that would have been realized without use of the storage resources due to the RES generation is subtracted.
}

proposed allocation mechanism (i.e., columns 5 and 6). In the first three columns, $\pi_{i}^{\text {op }}$ is shown for the case where the players are each allocated $100 \%$ of the storage resources. Column four indicates $\pi_{i}^{\mathrm{op}}$ in case each player is awarded a fixed share equal to one-third of the storage resources for the entire year. Since the players are assumed to be price-takers in their respective markets (i.e., dayahead electricity and real-time balancing market), one may expect that $\pi_{i}^{\mathrm{op}}$ is equal to one-third of $\pi_{i}^{\mathrm{op}}$ following a $100 \%$ allocation to the respective player. Although this is the case for player $a$, this is not the case for player $p$ (42.5\%) and player $r(61.5 \%)$ as their actions are limited by $G_{p, h}$ and $L_{r}^{\max }$ in the provided case study. Column five shows $\pi_{i}^{\text {op }}$ when assuming a daily organized auction with a single market-clearing, i.e., daily allocated (dis)charge power and storage capacity rights, while column six is based on a daily organized auction with hourly market-clearings. Fig. 4 shows that the auction-based allocations lead to a higher total realized operating profit $\sum_{i \in \mathbb{}} \pi_{i}^{\mathrm{op}}$, with shorter time frames for the marketclearings performing better. The latter ensures that the limited storage resources are allocated to the most valuable services at each point in time.

Table 3 shows $\pi_{i}^{\mathrm{op}}, \beta_{i}$, and $\pi_{i}$ for the different players. The revenue collected through the auctioning of the (dis)charge power and storage capacity rights is indicated by $\sum_{i \in \mathbb{1}} \beta_{i}$. The price of an auctioned right (i.e., $\mu_{h}^{\mathrm{c}}, \mu_{h}^{\mathrm{d}}, \mu_{h}^{\mathrm{e}}$ for hourly allocations and $\mu^{\mathrm{c}}, \mu^{\mathrm{d}}, \mu^{\mathrm{e}}$ for single allocations per auction) only takes on a nonzero value when the inequality constraint representing the limited availability of the storage resource subject to the constraint is binding (i.e., (A.4a)-(A.4c) for hourly allocations and (A.8a)-(A.8c) for single allocations per auction). In case the price is nonzero, it takes on the willingness-to-pay of the demand bid of the marginally cleared player for the respective resource. As such, the zero profit $\pi_{d}$ and close-to-zero profit $\pi_{p}$ indicate that when these players' bids to obtain storage rights are accepted they represent the marginally cleared bids. This is similar to the situation in electricity markets, where the player of the marginally cleared demand bid pays as much as he values the consumption of electric power during that market period. Contrarily, the positive profit $\pi_{r}$ shows that its bids to obtain storage rights not always represent the marginally cleared bid. This can be explained by the large price spreads at the real-time market compared to the dayahead market, through which player $r$ values the use of storage resources higher, and because the inequality constraints are not binding when he is the only player that contracts storage resources as its (dis)charge actions are limited by $L_{r}^{\max }$. In the former case player $r$ pays the lower willingness-to-pay of one of the other cleared players, while in the latter case the price of the right of the storage resource subject to the constraint is zero. 


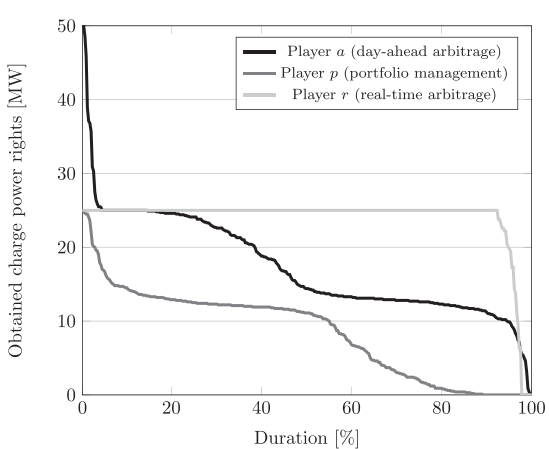

(a) Daily allocation of charge power rights.

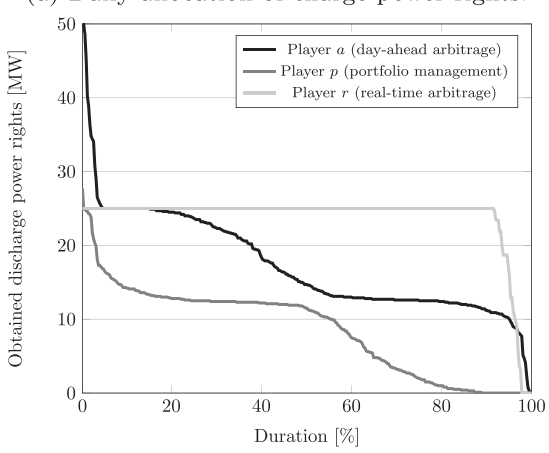

(c) Daily allocation of discharge power rights.

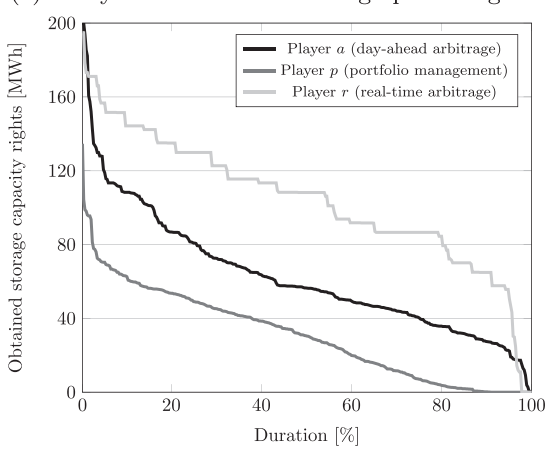

(e) Daily allocation of storage capacity rights

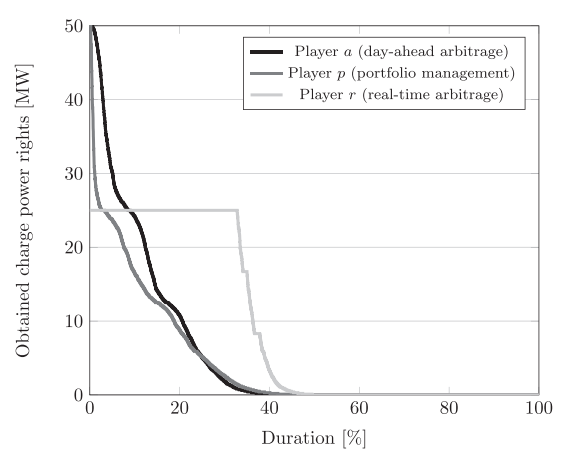

(b) Hourly allocation of charge power rights.

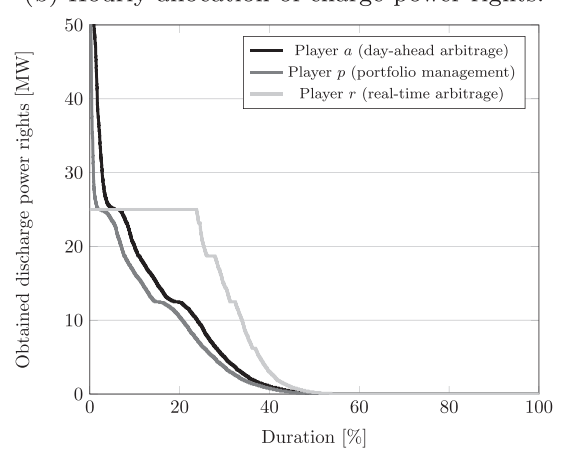

(d) Hourly allocation of discharge power rights.

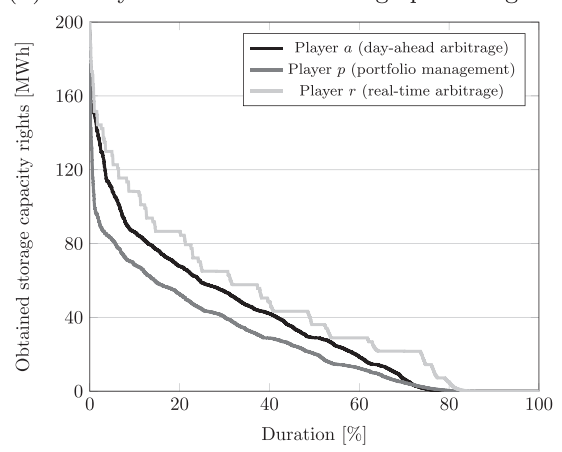

(f) Hourly allocation of storage capacity rights.

Fig. 5. Allocation of physical storage rights in a daily auction with daily (a), (c), (e), and hourly (b), (d), (f) market-clearings, 2014.

Contrary to this illustrative case study, as more players participate in such an auction, and more applications are considered, these situations will occur less frequently. As such, the revenue collected through the auctioning of storage rights will converge to the total captured value in the electricity market more closely.

Fig. 5 illustrates the allocation of the auctioned (dis)charge power and storage capacity rights for a daily auction with daily (a, $c$ and e) and hourly (b, d and f) market-clearings for 2014. As for price-taking players with perfect foresight the overall daily value of storage resources is likely to be higher for arbitraging real-time imbalance prices than arbitraging day-ahead electricity prices, due to the larger and more frequent price spreads, player $a$ and player $p$ do not often get the opportunity to use more than $25 \mathrm{MW}$ in the daily allocation case. However, for some hours of the day they might actually have a higher willingness-to-pay and thus the storage resources would be valorized at a higher value. Therefore, when using more frequent market-clearings (i.e., with shorter durations), the storage resources are allocated more efficiently to the time-varying most valuable services, resulting in a higher total storage value.

\section{Conclusions}

Electricity storage has the ability to compensate temporary power surpluses and shortages by decoupling the generation of electric energy from its consumption over time, thereby meeting increased flexibility needs. However, market participants are only incentivized to invest in new flexible resources when the investment is profitable. As this may not be the case when only considering a single or a few storage services, maximizing the value of electricity storage requires the aggregation of multiple value streams in a single operating strategy.

As such, this article proposes a new storage business model based on multiple simultaneously considered revenue streams, in which the applications or even the player that the storage resources will serve at a certain moment in time are not predefined. This can be accomplished by the development of a platform where periodical auctions with sequential marketclearings take place to allocate physical storage rights to use (dis)charge power capacities and energy storage capacity. These auctions allow storage owners to commercialize their resources over different applications, while players looking for additional 
flexibility can obtain this on a short-term basis. Alternatively, through such an allocation mechanism players can effectively share storage resources. The mechanism allocates the resources to the most valuable application for each market-clearing, based on the players' willingness-to-pay, which directly relates to the improvement of their objective value from a marginal improvement of the respective storage resource.

Players may be incentivized to participate in such a mechanism to share the investment cost, mitigate the associated risk, exploit economies of scale, overcome regulatory barriers, and merge timevarying and player-dependent flexibility needs. In addition, this may include positive effects for the system as well, as limited storage resources are allocated to the most valuable services at each point in time and the strategic operation of storage resources is likely to occur less frequently due to the introduced competition.

Future work includes the comparison of the explicit auctioning of storage resources through physical storage rights to a centralized operation of storage with implicit auctioning and to financial storage rights. In addition, future research includes the analysis of different design parameters (e.g., lead times between the auction and physical delivery, allocation horizon), as well as the accommodation of flexible consumption processes in this flexibility platform because of the similarities with electricity storage plants (e.g., limited duration).

\section{Acknowledgments}

The authors would like to thank Benjamin F. Hobbs for his feedback on a preliminary version of this work, and to co-host Tom Brijs at The Johns Hopkins University. The authors would also like to thank Cedric De Jonghe and Frederik Geth for valuable comments, and Research Foundation Flanders (FWO) for providing Tom Brijs with a travel grant for an extended research visit at The Johns Hopkins University.

\section{Appendix A}

First the MCP formulation for a periodically organized auction with hourly market-clearings for the shared storage resources is presented. The KKT conditions of player $a$ are (A.1a)-(A.1j), while those of player $p$ are (A.2a)-(A.2m), and finally the KKT conditions of player $r$ are (A.3a)-(A.3k). The shared constraints are represented by (A.4a)-(A.4c) in the MCP formulation:

$$
\begin{aligned}
& 0 \leq \lambda_{h}^{\mathrm{da}} /|\mathbb{H}|+T^{\mathrm{h}} \cdot \gamma_{a, h}^{\mathrm{e}} \cdot \eta^{\mathrm{c}}+\tau_{a, h}^{\mathrm{c}} \perp p_{a, h}^{\mathrm{c}} \geq 0, \quad \forall h \in \mathbb{U}, \\
& 0 \leq-\lambda_{h}^{\mathrm{da}} /|\mathbb{H}|-T^{\mathrm{h}} \cdot \gamma_{a, h}^{\mathrm{e}} / \eta^{\mathrm{d}}+\tau_{a, h}^{\mathrm{d}} \perp p_{a, h}^{\mathrm{d}} \geq 0, \quad \forall h \in \mathbb{U}, \\
& 0 \leq-\gamma_{a, h}^{\mathrm{e}}+\gamma_{a, h+1}^{\mathrm{e}}+\tau_{a, h}^{\mathrm{e}} \perp e_{a, h} \geq 0, \quad \forall h \in \mathbb{U}, \\
& 0 \leq-\tau_{a, h}^{\mathrm{c}}+\mu_{h}^{\mathrm{c}} \perp p_{a, h}^{\mathrm{c}, \max } \geq 0, \quad \forall h \in \mathbb{U}, \\
& 0 \leq-\tau_{a, h}^{\mathrm{d}}+\mu_{h}^{\mathrm{d}} \perp p_{a, h}^{\mathrm{d}, \max } \geq 0, \quad \forall h \in \mathbb{U}, \\
& 0 \leq-\tau_{a, h}^{\mathrm{e}}+\mu_{h}^{\mathrm{e}} \perp e_{a, h}^{\max } \geq 0, \quad \forall h \in \mathbb{U}, \\
& 0=-e_{a, h}+e_{a, h-1}+T^{\mathrm{h}} \cdot\left(p_{a, h}^{\mathrm{c}} \cdot \eta^{\mathrm{c}}-p_{a, h}^{\mathrm{d}} / \eta^{\mathrm{d}}\right), \\
& \gamma_{a, h}^{\mathrm{e}} \in \mathbb{R}, \quad \forall h \in \mathbb{H}, \\
& 0 \leq p_{a, h}^{\mathrm{c}, \max }-p_{a, h}^{\mathrm{c}} \perp \tau_{a, h}^{\mathrm{c}} \geq 0, \quad \forall h \in \mathbb{U},
\end{aligned}
$$

$$
\begin{aligned}
& 0 \leq p_{a, h}^{\mathrm{d}, \max }-p_{a, h}^{\mathrm{d}} \perp \tau_{a, h}^{\mathrm{d}} \geq 0, \quad \forall h \in \mathbb{H}, \\
& 0 \leq e_{a, h}^{\max }-e_{a, h} \perp \tau_{a, h}^{\mathrm{e}} \geq 0, \quad \forall h \in \mathbb{H}, \\
& 0 \leq \gamma_{p, h}^{\mathrm{g}}+T^{\mathrm{h}} \cdot \gamma_{p, h}^{\mathrm{e}} \cdot \eta^{\mathrm{c}}+\tau_{p, h}^{\mathrm{c}} \perp p_{p, h}^{\mathrm{c}} \geq 0, \quad \forall h \in \mathbb{U}, \\
& 0 \leq-\lambda_{h}^{\mathrm{da}} /|\mathbb{H}|-T^{\mathrm{h}} \cdot \gamma_{p, h}^{\mathrm{e}} / \eta^{\mathrm{d}}+\tau_{p, h}^{\mathrm{d}} \perp p_{p, h}^{\mathrm{d}} \geq 0, \quad \forall h \in \mathbb{H}, \\
& 0 \leq-\gamma_{p, h}^{\mathrm{e}}+\gamma_{p, h+1}^{\mathrm{e}}+\tau_{p, h}^{\mathrm{e}} \perp e_{p, h} \geq 0, \quad \forall h \in \mathbb{U}, \\
& 0 \leq-\lambda_{h}^{\mathrm{da}} /|\mathbb{H}|+\gamma_{p, h}^{\mathrm{g}} \perp p_{p, h}^{\mathrm{g}} \geq 0, \quad \forall h \in \mathbb{U}, \\
& 0 \leq \gamma_{p, h}^{\mathrm{g}} \perp p_{p, h}^{\mathrm{l}} \geq 0, \quad \forall h \in \mathbb{U}, \\
& 0 \leq-\tau_{p, h}^{\mathrm{c}}+\mu_{h}^{\mathrm{c}} \perp p_{p, h}^{\mathrm{c}, \max } \geq 0, \quad \forall h \in \mathbb{H}, \\
& 0 \leq-\tau_{p, h}^{\mathrm{d}}+\mu_{h}^{\mathrm{d}} \perp p_{p, h}^{\mathrm{d}, \max } \geq 0, \quad \forall h \in \mathbb{H}, \\
& 0 \leq-\tau_{p, h}^{\mathrm{e}}+\mu_{h}^{\mathrm{e}} \perp e_{p, h}^{\max } \geq 0, \quad \forall h \in \mathbb{M}, \\
& 0=-G_{p, h}+p_{p, h}^{\mathrm{c}}+p_{p, h}^{\mathrm{g}}+p_{p, h}^{\mathrm{l}}, \gamma_{p, h}^{\mathrm{g}} \in \mathbb{R}, \quad \forall h \in \mathbb{H}, \\
& 0=-e_{p, h}+e_{p, h-1}+T^{\mathrm{h}} \cdot\left(p_{p, h}^{\mathrm{c}} \cdot \eta^{\mathrm{c}}-p_{p, h}^{\mathrm{d}} / \eta^{\mathrm{d}}\right), \\
& \gamma_{p, h}^{\mathrm{e}} \in \mathbb{R}, \quad \forall h \in \mathbb{H}, \\
& 0 \leq p_{p, h}^{c, \max }-p_{p, h}^{\mathrm{c}} \perp \tau_{p, h}^{\mathrm{c}} \geq 0, \quad \forall h \in \mathbb{U}, \\
& 0 \leq p_{p, h}^{\mathrm{d}, \max }-p_{p, h}^{\mathrm{d}} \perp \tau_{p, h}^{\mathrm{d}} \geq 0, \quad \forall h \in \mathbb{U}, \\
& 0 \leq e_{p, h}^{\max }-e_{p, h} \perp \tau_{p, h}^{\mathrm{e}} \geq 0, \quad \forall h \in \mathbb{U},
\end{aligned}
$$

$0 \leq \lambda_{h}^{\mathrm{rt}} /|\mathbb{H}|+T^{\mathrm{h}} \cdot \gamma_{r, h}^{\mathrm{e}} \cdot \eta^{\mathrm{c}}+\gamma_{r, h}^{\mathrm{l}}+\tau_{r, h}^{\mathrm{c}} \perp p_{r, h}^{\mathrm{c}} \geq 0, \quad \forall h \in \mathbb{H}$,

$0 \leq-\lambda_{h}^{\mathrm{da}} /|\mathbb{M}|-T^{\mathrm{h}} \cdot \gamma_{r, h}^{\mathrm{e}} / \eta^{\mathrm{d}}+\gamma_{r, h}^{\mathrm{l}}+\tau_{r, h}^{\mathrm{d}} \perp p_{r, h}^{\mathrm{d}} \geq 0, \quad \forall h \in \mathbb{M}$,

$0 \leq-\gamma_{r, h}^{\mathrm{e}}+\gamma_{r, h+1}^{\mathrm{e}}+\tau_{r, h}^{\mathrm{e}} \perp e_{r, h} \geq 0, \quad \forall h \in \mathbb{U}$,

$0 \leq-\tau_{r, h}^{\mathrm{c}}+\mu_{h}^{\mathrm{c}} \perp p_{r, h}^{\mathrm{c}, \max } \geq 0, \quad \forall h \in \mathbb{M}$,

$0 \leq-\tau_{r, h}^{\mathrm{d}}+\mu_{h}^{\mathrm{d}} \perp p_{r, h}^{\mathrm{d}, \max } \geq 0, \quad \forall h \in \mathbb{U}$,

$0 \leq-\tau_{r, h}^{\mathrm{e}}+\mu_{h}^{\mathrm{e}} \perp e_{r, h}^{\max } \geq 0, \quad \forall h \in \mathbb{U}$,

$0 \leq L_{r}^{\max }-p_{r, h}^{\mathrm{c}}-p_{r, h}^{\mathrm{d}} \perp \gamma_{r, h}^{\mathrm{l}} \geq 0, \quad \forall h \in \mathbb{U}$,

$0=-e_{r, h}+e_{r, h-1}+T^{\mathrm{h}} \cdot\left(p_{r, h}^{\mathrm{c}} \cdot \eta^{\mathrm{c}}-p_{r, h}^{\mathrm{d}} / \eta^{\mathrm{d}}\right)$, $\gamma_{r, h}^{\mathrm{e}} \in \mathbb{R}, \quad \forall h \in \mathbb{H}$,

$0 \leq p_{r, h}^{c, m a x}-p_{r, h}^{c} \perp \tau_{r, h}^{c} \geq 0, \quad \forall h \in \mathbb{U}$,

$0 \leq p_{r, h}^{\mathrm{d}, \max }-p_{r, h}^{\mathrm{d}} \perp \tau_{r, h}^{\mathrm{d}} \geq 0, \quad \forall h \in \mathbb{H}$,

$0 \leq e_{r, h}^{\max }-e_{r, h} \perp \tau_{r, h}^{\mathrm{e}} \geq 0, \quad \forall h \in \mathbb{U}$,

$0 \leq P^{c, \max }-p_{a, h}^{c, \max }-p_{p, h}^{c, \max }-p_{r, h}^{c, \text { max }} \perp \mu_{h}^{c} \geq 0, \quad \forall h \in \mathbb{U}$,

$0 \leq P^{\mathrm{d}, \max }-p_{a, h}^{\mathrm{d}, \text { max }}-p_{p, h}^{\mathrm{d}, \text { max }}-p_{r, h}^{\mathrm{d}, \mathrm{max}} \perp \mu_{h}^{\mathrm{d}} \geq 0, \quad \forall h \in \mathbb{U}$, 
$0 \leq E^{\max }-e_{a, h}^{\max }-e_{p, h}^{\max }-e_{r, h}^{\max } \perp \mu_{h}^{\mathrm{e}} \geq 0, \quad \forall h \in \mathbb{U}$.

(A.4c)

Second the MCP formulation for a less dynamic periodically organized auction (e.g., daily, weekly, monthly) including a single market-clearing, i.e., allocation, for each of the shared resources for the entire period (e.g., day, week, month) is presented. In this case the KKT conditions of player $a$ are (A.5a)-(A.5j), of player $p$ are (A.6a)-(A.6m), and finally of player $r$ are (A.7a)-(A.7k). The shared constraints are included through (A.8a)-(A.8c) in the MCP formulation:

$$
\begin{aligned}
& 0 \leq \lambda_{h}^{\mathrm{da}} /|\mathbb{H}|+T^{\mathrm{h}} \cdot \gamma_{a, h}^{\mathrm{e}} \cdot \eta^{\mathrm{c}}+\tau_{a, h}^{\mathrm{c}} \perp p_{a, h}^{\mathrm{c}} \geq 0, \quad \forall h \in \mathbb{U}, \\
& 0 \leq-\lambda_{h}^{\mathrm{da}} /|\mathbb{H}|-T^{\mathrm{h}} \cdot \gamma_{a, h}^{\mathrm{e}} / \eta^{\mathrm{d}}+\tau_{a, h}^{\mathrm{d}} \perp p_{a, h}^{\mathrm{d}} \geq 0, \quad \forall h \in \mathbb{U}, \\
& 0 \leq-\gamma_{a, h}^{\mathrm{e}}+\gamma_{a, h+1}^{\mathrm{e}}+\tau_{a, h}^{\mathrm{e}} \perp e_{a, h} \geq 0, \quad \forall h \in \mathbb{U}, \\
& 0 \leq \sum_{h \in \mathbb{H}}\left(-\tau_{a, h}^{\mathrm{c}}\right)+\mu^{\mathrm{c}} \perp p_{a}^{\mathrm{c}, \max } \geq 0, \\
& 0 \leq \sum_{h \in \mathbb{H}}\left(-\tau_{a, h}^{\mathrm{d}}\right)+\mu^{\mathrm{d}} \perp p_{a}^{\mathrm{d}, \max } \geq 0, \\
& 0 \leq \sum_{h \in \mathbb{H}}\left(-\tau_{a, h}^{\mathrm{e}}\right)+\mu^{\mathrm{e}} \perp e_{a}^{\max } \geq 0, \\
& 0=-e_{a, h}+e_{a, h-1}+T^{\mathrm{h}} \cdot\left(p_{a, h}^{\mathrm{c}} \cdot \eta^{\mathrm{c}}-p_{a, h}^{\mathrm{d}} / \eta^{\mathrm{d}}\right), \\
& \gamma_{a, h}^{\mathrm{e}} \in \mathbb{R}, \quad \forall h \in \mathbb{H}, \\
& 0 \leq p_{a}^{\mathrm{c}, \max }-p_{a, h}^{\mathrm{c}} \perp \tau_{a, h}^{\mathrm{c}} \geq 0, \quad \forall h \in \mathbb{H}, \\
& 0 \leq p_{a}^{\mathrm{d}, \max }-p_{a, h}^{\mathrm{d}} \perp \tau_{a, h}^{\mathrm{d}} \geq 0, \quad \forall h \in \mathbb{U}, \\
& 0 \leq e_{a}^{\max }-e_{a, h} \perp \tau_{a, h}^{\mathrm{e}} \geq 0, \quad \forall h \in \mathbb{H},
\end{aligned}
$$

$0 \leq \gamma_{p, h}^{\mathrm{g}}+T^{\mathrm{h}} \cdot \gamma_{p, h}^{\mathrm{e}} \cdot \eta^{\mathrm{c}}+\tau_{p, h}^{\mathrm{c}} \perp p_{p, h}^{\mathrm{c}} \geq 0, \quad \forall h \in \mathbb{M}$,

$0 \leq-\lambda_{h}^{\mathrm{da}} /|\mathbb{H}|-T^{\mathrm{h}} \cdot \gamma_{p, h}^{\mathrm{e}} / \eta^{\mathrm{d}}+\tau_{p, h}^{\mathrm{d}} \perp p_{p, h}^{\mathrm{d}} \geq 0, \quad \forall h \in \mathbb{H}$,

$0 \leq-\gamma_{p, h}^{\mathrm{e}}+\gamma_{p, h+1}^{\mathrm{e}}+\tau_{p, h}^{\mathrm{e}} \perp e_{p, h} \geq 0, \quad \forall h \in \mathbb{U}$,

$0 \leq-\lambda_{h}^{\mathrm{da}} /|\mathbb{W}|+\gamma_{p, h}^{\mathrm{g}} \perp p_{p, h}^{\mathrm{g}} \geq 0, \quad \forall h \in \mathbb{M}$,

$0 \leq \gamma_{p, h}^{\mathrm{g}} \perp p_{p, h}^{\mathrm{l}} \geq 0, \quad \forall h \in \mathbb{U}$,

$0 \leq \sum_{h \in \mathbb{W}}\left(-\tau_{p, h}^{\mathrm{c}}\right)+\mu^{\mathrm{c}} \perp p_{p}^{\mathrm{c}, \max } \geq 0$,

$$
0 \leq \sum_{h \in \mathbb{\sharp}}\left(-\tau_{p, h}^{\mathrm{d}}\right)+\mu^{\mathrm{d}} \perp p_{p}^{\mathrm{d}, \max } \geq 0,
$$

$$
0 \leq \sum_{h \in \mathbb{H}}\left(-\tau_{p, h}^{\mathrm{e}}\right)+\mu^{\mathrm{e}} \perp e_{p}^{\max } \geq 0,
$$

$0=-G_{p, h}+p_{p, h}^{\mathrm{c}}+p_{p, h}^{\mathrm{g}}+p_{p, h}^{\mathrm{l}}, \gamma_{p, h}^{\mathrm{g}} \in \mathbb{R}, \quad \forall h \in \mathbb{U}$,

$0=-e_{p, h}+e_{p, h-1}+T^{\mathrm{h}} \cdot\left(p_{p, h}^{\mathrm{c}} \cdot \eta^{\mathrm{c}}-p_{p, h}^{\mathrm{d}} / \eta^{\mathrm{d}}\right)$,

$\gamma_{p, h}^{\mathrm{e}} \in \mathbb{R}, \quad \forall h \in \mathbb{H}$,

$0 \leq p_{p}^{\mathrm{c}, \max }-p_{p, h}^{\mathrm{c}} \perp \tau_{p, h}^{\mathrm{c}} \geq 0, \quad \forall h \in \mathbb{U}$,

$$
\begin{aligned}
& 0 \leq p_{p}^{\mathrm{d}, \max }-p_{p, h}^{\mathrm{d}} \perp \tau_{p, h}^{\mathrm{d}} \geq 0, \quad \forall h \in \mathbb{U}, \\
& 0 \leq e_{p}^{\max }-e_{p, h} \perp \tau_{p, h}^{\mathrm{e}} \geq 0, \quad \forall h \in \mathbb{M}, \\
& 0 \leq \lambda_{h}^{\mathrm{rt}} /|\mathbb{U}|+T^{\mathrm{h}} \cdot \gamma_{r, h}^{\mathrm{e}} \cdot \eta^{\mathrm{c}}+\gamma_{r, h}^{\mathrm{l}}+\tau_{r, h}^{\mathrm{c}} \perp p_{r, h}^{\mathrm{c}} \geq 0, \quad \forall h \in \mathbb{U} \\
& 0 \leq-\lambda_{h}^{\mathrm{rt}} /|\mathbb{H}|-T^{\mathrm{h}} \cdot \gamma_{r, h}^{\mathrm{e}} / \eta^{\mathrm{d}}+\gamma_{r, h}^{\mathrm{l}}+\tau_{r, h}^{\mathrm{d}} \perp p_{r, h}^{\mathrm{d}} \geq 0, \quad \forall h \in \mathbb{H}, \\
& 0 \leq-\gamma_{r, h}^{\mathrm{e}}+\gamma_{r, h+1}^{\mathrm{e}}+\tau_{r, h}^{\mathrm{e}} \perp e_{r, h} \geq 0, \quad \forall h \in \mathbb{U}, \\
& 0 \leq \sum_{h \in \mathbb{H}}\left(-\tau_{r, h}^{\mathrm{c}}\right)+\mu^{\mathrm{c}} \perp p_{r}^{\mathrm{c}, \max } \geq 0, \\
& 0 \leq \sum_{h \in \mathbb{H}}\left(-\tau_{r, h}^{\mathrm{d}}\right)+\mu^{\mathrm{d}} \perp p_{r}^{\mathrm{d}, \max } \geq 0, \\
& 0 \leq \sum_{h \in \mathbb{H}}\left(-\tau_{r, h}^{\mathrm{e}}\right)+\mu^{\mathrm{e}} \perp e_{r}^{\max } \geq 0, \\
& 0 \leq L_{r}^{\max }-p_{r, h}^{\mathrm{c}}-p_{r, h}^{\mathrm{d}} \perp \gamma_{r, h}^{\mathrm{l}} \geq 0, \quad \forall h \in \mathbb{U}, \\
& 0=-e_{r, h}+e_{r, h-1}+T^{\mathrm{h}} \cdot\left(p_{r, h}^{\mathrm{c}} \cdot \eta^{\mathrm{c}}-p_{r, h}^{\mathrm{d}} / \eta^{\mathrm{d}}\right) \\
& \gamma_{r, h}^{\mathrm{e}} \in \mathbb{R}, \quad \forall h \in \mathbb{U}, \\
& 0 \leq p_{r}^{c, m a x}-p_{r, h}^{c} \perp \tau_{r, h}^{c} \geq 0, \quad \forall h \in \mathbb{U}, \\
& 0 \leq p_{r}^{\mathrm{d}, \max }-p_{r, h}^{\mathrm{d}} \perp \tau_{r, h}^{\mathrm{d}} \geq 0, \quad \forall h \in \mathbb{U}, \\
& 0 \leq e_{r}^{\max }-e_{r, h} \perp \tau_{r, h}^{\mathrm{e}} \geq 0, \quad \forall h \in \mathbb{H}, \\
& 0 \leq P^{c, \text { max }}-p_{a}^{c, \text { max }}-p_{p}^{c, \text { max }}-p_{r}^{c, \text { max }} \perp \mu^{\mathrm{c}} \geq 0, \\
& 0 \leq P^{\mathrm{d}, \max }-p_{a}^{\mathrm{d}, \max }-p_{p}^{\mathrm{d}, \max }-p_{r}^{\mathrm{d}, \max } \perp \mu^{\mathrm{d}} \geq 0, \\
& 0 \leq E^{\max }-e_{a}^{\max }-e_{p}^{\max }-e_{r}^{\max } \perp \mu^{\mathrm{e}} \geq 0 .
\end{aligned}
$$

\section{References}

[1] T. Brijs, K. De Vos, C. De Jonghe, R. Belmans, Statistical analysis of negative prices in European balancing markets, Renew. Energy 80 (2015) 53-60, http://dx.doi.org/ 10.1016/j.renene.2015.01.059.

[2] E. Lannoye, D. Flynn, M. O'Malley, Evaluation of power system flexibility, IEEE Trans. Power Syst. 27 (2) (2012) 922-931, http://dx.doi.org/10.1109/TPWRS.2011.2177280.

[3] J. Ma, V. Silva, R. Belhomme, D.S. Kirschen, L.F. Ochoa, Evaluating and planning flexibility in sustainable power systems, IEEE Trans. Sustain. Energy 4 (1) (2013) 200-209, http://dx.doi.org/10.1109/PESMG.2013.6672221.

[4] X. He, E. Delarue, W. D'haeseleer, J.-M. Glachant, A novel business model for aggregating the values of electricity storage, Energy Policy 39 (3) (2011) 15751585, http://dx.doi.org/10.1016/j.enpol.2010.12.033.

[5] R. Moreno, R. Moreira, G. Strbac, A MILP model for optimising multi-service portfolios of distributed energy storage, Appl. Energy 137 (2015) 554-566, http:// dx.doi.org/10.1016/j.apenergy.2014.08.080.

[6] D. Pudjianto, M. Aunedi, P. Djapic, G. Strbac, Whole-systems assessment of the value of energy storage in low-carbon electricity systems, IEEE Trans. Smart Grid 5 (2) (2014) 1098-1109, http://dx.doi.org/10.1109/TSG.2013.2282039.

[7] X. Xi, R. Sioshansi, V. Marano, A stochastic dynamic programming model for cooptimization of distributed energy storage, Energy Syst. 5 (3) (2013) 475-505, http://dx.doi.org/10.1007/s12667-013-0100-6.

[8] A. Castillo, D.F. Gayme, Grid-scale energy storage applications in renewable energy integration: a survey, Energy Convers. Manag. 87 (2014) 885-894, http://dx.doi.org/10.1016/j.enconman.2014.07.063.

[9] A. Akhil, G. Huff, A. Currier, B. Kaun, D. Rastler, S.B. Chen, A. Cotter, D. Bradshaw, W Gauntlett, DOE /EPRI 2013 Electricity Storage Handbook in Collaboration with NRECA, Tech. rep., Sandia National Laboratories, Albuquerque, NM/Livermore, CA, 2013, http://www.sandia.gov/ess/publications/SAND2013-5131.pdf.

[10] J. Eyer, G. Corey, Energy storage for the electricity grid: Benefits and market potential assessment guide. Tech. Rep. February, Sandia National Laboratories, Albuquerque, NM/Livermore, CA, 2010, http://www.sandia.gov/ess/publications/ SAND2010-0815.pdf. 
[11] P. Denholm, E. Ela, B. Kirby, M. Milligan, The role of energy storage with renewable electricity generation. Tech. Rep. January, National Renewable Energy Laboratory, Golden, CO, 2010, http://www.nrel.gov/docs/fy10osti/47187.pdf.

[12] E. Drury, P. Denholm, R. Sioshansi, The value of compressed air energy storage in energy and reserve markets, Energy 36 (8) (2011) 4959-4973, http://dx.doi.org/ 10.1016/j.energy.2011.05.041.

[13] R. Sioshansi, Welfare impacts of electricity storage and the implications of ownership structure, Energy J. 31 (2) (2009) 173-198, http://dx.doi.org/10.5547/ ISSN0195-6574-EJ-Vol31-No2-7.

[14] F. Geth, T. Brijs, J. Kathan, J. Driesen, R. Belmans, An overview of large-scale stationary electricity storage plants in Europe: current status and new developments, Renew. Sustain. Energy Rev. 52 (2015) 1212-1227, http://dx.doi.org/ 10.1016/j.rser.2015.07.145.

[15] M. Abdurrahman, S. Baker, B. Keshavamurthy, M. Jacobs, Energy storage as a transmission asset. Tech. rep., PJM Interconnection and Xtreme Power, 2012, http://www.pjm.com/\{ $\} /$ media/markets-ops/advanced-tech-pilots/ xtreme-power-storage-as-transmission.ashx.

[16] L. Meeus, Implicit auctioning on the Kontek cable: third time lucky? Energy Econ. 33 (3) (2011) 413-418, http://dx.doi.org/10.1016/j.eneco.2010.10.008.

[17] T. Kristiansen, An assessment of the Danish-German cross-border auctions, Energy Policy 35 (6) (2007) 3369-3382, http://dx.doi.org/10.1016/j.enpol.2006. 11.023.

[18] J.A. Taylor, Financial storage rights, IEEE Trans. Power Syst. 30 (2) (2015) 9971005, http://dx.doi.org/10.1109/TPWRS.2014.2339016.

[19] D. Munoz-Alvarez, E. Bitar, Financial storage rights: definition and basic properties, in: North American Power Symposium, IEEE, Pullmann, WA, USA, (2014), pp. 1-6, http://dx.doi.org/10.1109/NAPS.2014.6965443.
[20] R.P. O'Neill, U. Helman, B.F. Hobbs, W.R. Stewart, M.H. Rothkopf, A joint energy and transmission rights auction: proposal and properties, IEEE Trans. Power Syst. 17 (4) (2002) 1058-1067, http://dx.doi.org/10.1109/TPWRS.2002.804978.

[21] T. Kristiansen, J. Rosellón, Merchant electricity transmission expansion: a European case study, Energy 35 (10) (2010) 4107-4115, http://dx.doi.org/10.1016/ j.energy.2010.06.025.

[22] J. Nash, Non-cooperative games, Ann. Math. 54 (2) (1951) 286-295.

[23] P.T. Harker, Generalized Nash games and quasi-variational inequalities, Eur. J. Oper. Res. 54 (1) (1991) 81-94, http://dx.doi.org/10.1016/0377-2217(91)90325-P.

[24] G. Oggioni, Y. Smeers, E. Allevi, S. Schaible, A Generalized Nash equilibrium model of market coupling in the European power system, Netw. Spat. Econ. 12 (4) (2012) 503-560, http://dx.doi.org/10.1007/s11067-011-9166-7.

[25] I. Abada, S. Gabriel, V. Briat, O. Massol, A Generalized Nash-Cournot model for the northwestern European natural gas markets with a fuel substitution demand function: the GaMMES model, Netw. Spat. Econ. 13 (1) (2013) 1-42, http:// dx.doi.org/10.1007/s11067-012-9171-5.

[26] F. Facchinei, A. Fischer, V. Piccialli, On Generalized Nash games and variational inequalities, Oper. Res. Lett. 35 (2) (2007) 159-164, http://dx.doi.org/10.1016/ j.orl.2006.03.004.

[27] S.A. Gabriel, A.J. Conejo, J.D. Fuller, B.F. Hobbs, C. Ruiz, Complementarity Modeling in Energy Markets, Springer, New York, USA, 2013, http://dx.doi.org/10.1007/ 978-1-4419-6123-5.

[28] M.C. Ferris, T.S. Munson, Complementarity problems in GAMS and the PATH solver, J. Econ. Dyn. Control 24 (2) (2000) 165-188, http://dx.doi.org/10.1016/ S0165-1889(98)00092-X

[29] Belpex, Historical Data, 2016, http://www.belpex.be/market-results/historical-data/.

[30] Elia, Data download page, 2016, http://www.elia.be/en/grid-data/data-download. 